\title{
Method of estimating the state of charge of a battery electric vehicle based on RS-SVM
}

\author{
Guocheng $\mathrm{Niu}^{1}$, Bongmei $\mathrm{Hu}^{2}$, and Jing $\mathrm{Bai}^{3}$, \\ ${ }^{1,2,3}$ College of Electrical and Information Engineering Beihua University, Jilin, 132021,China
}

Keywords: Rough set. Support vector machine.soc

\begin{abstract}
Charging, discharge, maintenance and energy management technology for electric vehicle power battery is relatively backward, An advanced and reasonable method is presented for battery state of charge( SOC ), used simple rough set attribute ,simplified battery charging related parameters, the simplified data is processed by using the support vector machine (SVM) to predict charged state of the battery, and the service life of the battery is lengthened .This paper has important research value.
\end{abstract}

\section{Introduction}

The phenomenon, the improper charging method leads to the car battery damage and the shortened life expectancy, remains commonplace. According to the battery state of charge (SOC) , the effective method is proposed to guide the charge, discharge and battery reasonable collocation for energy-saving the battery life increasing.

Affected by many factors such as power rate,battery temperature, self-discharge rate, battery different working rate and so on, It is difficult for conventional method to correctly predict battery state of charge( SOC ).

At present, the main detection methods of SOC, such as counting method, resistance method, open circuit voltage method and neural network method and so on. can not meet the various needs of the working state of the battery, the SOC estimation error is large. A new method is presented based on RS-SVM. The method consists of two main processes: Data reduction, processed battery charging history data based on the rough set theory, constructed a new learning sample and test sample, dynamically predicted the state of charge of battery based SVM.

\section{rough set and support vector machine}

Support vector machine (SVM) proposed by Vapnik , is a new machine learning method based on statistic learning theory - linear network ,is under the principle of structural risk minimization, According to the maximum classification interval output pattern recognition is developed, and the decision function is determined by the optimal classification hyper plane.However, it can't distinguish good data and bad data on processing to the training samples, thus, is likely to lower the SVM real-time prediction system.In order to solve this problem,preprocessed a large number of training sample data to eliminate redundant, useless information, high reliability training samples is provided for SVM, improved the efficiency of SVM prediction system. Rough set solved the problem.

Z.Pawlak pioneered the concept of rough set (RS), through the analysis of data uncertainty, and processing data by fuzzy knowledge.Attribute reduction and relative attribute reduction are a core of KDD.Rough sets [] can be expressed as: for the decision system $\mathrm{S}=(U, C \cup D, V, f)$, In the formula, domain $U=\{x 1, x 2 \ldots x n\}$ represents a collection of $n$ the research object; attribute set $\mathrm{A}=C \cup U(C \cup D=\phi), \mathrm{C}$ and $\mathrm{D}$ represent the condition attributes and the decision attributes; the attribute domain $\boldsymbol{V}=\underset{a_{i} \in A}{U} V_{a_{i}} ; f$ is the mapping function of attribute domain object. (U,A,f) is a decision table. 
Using the complementarity of rough sets and support vector machines,Prediction system of battery Soc based on RS-SVM is proposed, as shown in figure 1.

the preposition system

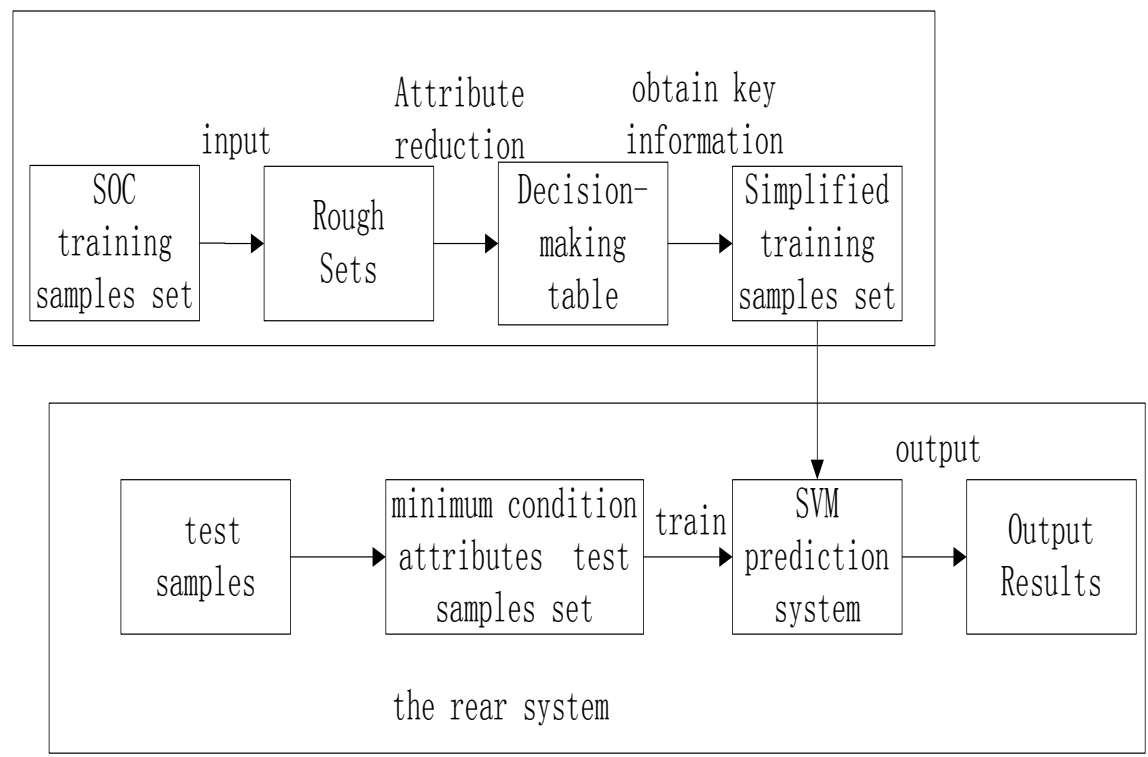

Figure 1 system structure diagram of battery SOC based on RS-SVM estimation

The system is composed of two parts, namely the front system and post system。Rough set network is the front system, includes the battery state of charge input sample, attribute reduction, decision table is established, the table data is obtained after simplification, is preprocessed data ,The SVM network is the post information recognition system, training on reduced samples to get the forecasting result.

\section{Improved reduction algorithm based on mutual information heuristic attribute}

For the decision system $\mathrm{S}, R \subseteq C$ The domain $\mathrm{U}$ is consistent in $\mathrm{C}$ compared with $\mathrm{D}$ $\left(\operatorname{POS}_{t}(D)=U\right), B \subseteq C$,Bis the relatively simple C,thus, $I(B, D)=I(C, D)$,that is, mutual information of reduced data remained unchanged.

When the condition attributes small affects decision attribute ,mutual information gain rate is larger, thus ,the selected decision attribute is not the most important, based on this, the attribute importance measurement can be defined as:

$$
\operatorname{SGF}(a, R, D)=(I(R \bigcup\{a\}, D)-I(R, D)) / I(a, D)
$$

This measurement method not only considers the increment of mutual information after adding attributes in the attribute $\mathrm{R}$, but also considers the mutual information of his own.

According to equation (2), the improved attribute reduction algorithm is established .This algorithm takes the core attributes of decision table as a starting point, selects successively,adds the largest non core condition attribute to the relative core set $\operatorname{SGF}(a, R, D)$, until a termination condition is satisfied $I(R, D)=I(C, D)$

Input: decision system $S=(U, C \cup D, V, f)$

Onput: Feature subset $\mathrm{R}$ of decision table information system。

(1) calculate attribute information between condition attributes and decision $I(C, D)$;

(2) calculate $R_{0}=\operatorname{Core}(C), I\left(R_{0}, D\right)$, if $I\left(R_{0}, D\right)=I(C, D)$,turn (5)else if continue,

(3)if $R=R_{0}, C=C-R$, calculate $\mathrm{C}$ according formula (2), and select $\operatorname{SGF}(a, R, D)$, form the greatest attribute set $a$; 
(4) $R=R \bigcup\{a\}$;

(5) $I(R, D)=I(C, D)$, terminate, otherwise turn step(3)。

\section{the parameters of support vector machine establish}

In the practical application,important parameters influencing SVM are: the penalty factor C and kernel function and its value.In the battery charging process, through comprehensivly analysis all parameters to judging battery SOC,Prediction method of battery SOC is described for the terminal voltage, $\mathrm{SOH} \%$ and residual capacity.Parameters distribution diagram of battery charging 20 times is shown in Figure 1。

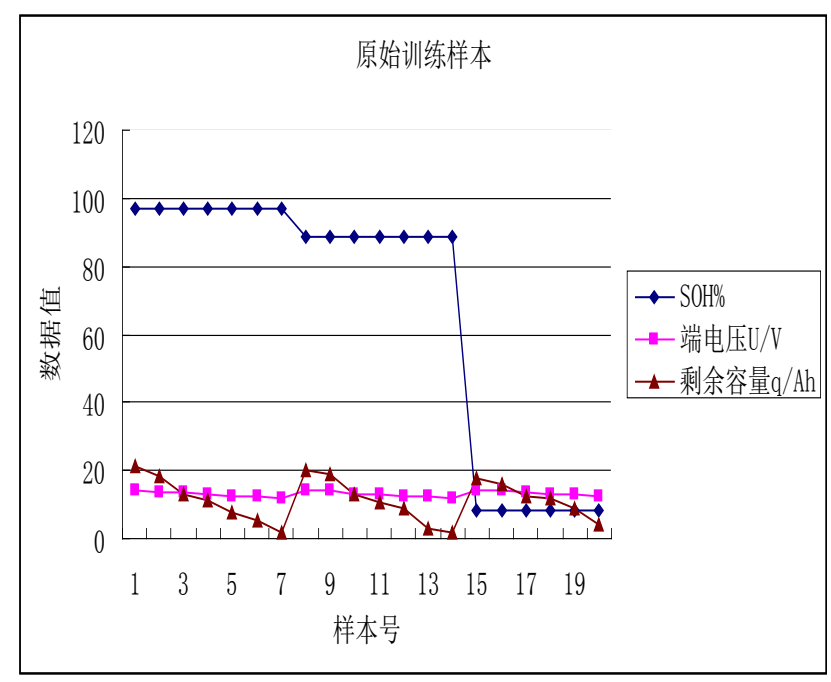

Figure 1 parameters distribution diagram of battery charging 20 times 。

the K-fold cross validation method was used. four kinds of kernel functions :

(1)Polynomial: $K\left(x, x_{i}\right)=\left[\left(x, x_{i}\right)+1\right]$

(2)G auss Function:

$$
K\left(x, x_{i}\right)=\exp \left\{-\frac{\left|x-x_{i}\right|^{2}}{2 \sigma^{2}}\right\}
$$

(3)R ad ialB asis Function (RBF) $K\left(x, x_{i}\right)=\exp \left(-\left|x-x_{i}\right|^{2} / \sigma^{2}\right)$

(4)S igm oid Function: $K\left(x, x_{i}\right)=\tanh \left[v\left(x \bullet x_{i}\right)+c\right]$

The training set consisted of the parameters data of 20 times of charging,was divided into 20 groups, used grid-search, GA and PSO to find the optimal combination of C and gamma value of the penalty parameter.The forecast effect produced is shown in table 1-3.

Table1 battery terminal voltage correlation coefficient $\mathrm{R}$

\begin{tabular}{cccc}
\hline & Grid-search/\% & GA/\% & PSO/\% \\
\hline Linear & 94.5265 & 96.7625 & 94.3018 \\
Polynomial & 86.3489 & 87.3487 & 86.7213 \\
RBF & 94.2431 & 97.7586 & 95.0456 \\
Sigmoid & 93.4325 & 95.7102 & 93.4709 \\
\hline
\end{tabular}


Table2 SOH\% correlation coefficient R

\begin{tabular}{cccc}
\hline & Grid-search/\% & GA/\% & PSO/\% \\
\hline Linear & 92.4789 & 98.4723 & 93.5238 \\
Polynomial & 86.8810 & 87.6813 & 84.6712 \\
RBF & 95.2213 & 97.3452 & 96.6724 \\
Sigmoid & 93.3647 & 96.7813 & 94.2672 \\
\hline
\end{tabular}

Table3 residual capacity correlation coefficient $\mathrm{R}$

\begin{tabular}{cccc}
\hline & Grid-search/\% & GA/\% & PSO/\% \\
\hline Linear & 91.3217 & 95.4017 & 92.4931 \\
Polynomial & 84.4078 & 86.0432 & 86.1089 \\
RBF & 93.0780 & 95.3452 & 95.2127 \\
Sigmoid & 92.2974 & 92.0431 & 93.3005 \\
\hline
\end{tabular}

Table 1 to table 3 shows that the epsilon-SVR-RBF-Genetic Algorithm model, called ERGA model, compared with other models, the prediction set the highest goodness of fit, the terminal voltage prediction accuracy reached $97.7586 \%$, the SOH prediction accuracy reaches $97.3452 \%$, the residual capacity reached $95.3452 \%$. In conclusion, The $\mathrm{SOH} \%$ and terminal voltage is less interfered in the normal charge and discharge cases, higher forecasting accuracy is reached,however,the residual capacity in the process of charge and discharge is influenced by the different charging method,which is low.

\section{Experimental results analysis}

Through the rough set method to remove redundant information, simplified the SVM complexity, improved the training speed. overcome noise sensitive defects of the rough set, makes the evaluation model has good performance. The experimental samples is shown in Table 4, the experimental and the predicted results and error analysis are shown in table 5.Training samples is In Table 4 , residual capacity is estimated using SR-SVM

Table 410 charging process samples

\begin{tabular}{|l|c|c|c|c|c|}
\hline Sample No. & 1 & 2 & 3 & 4 & 5 \\
\hline SOH\% & 96.8 & 96.8 & 96.8 & 96.8 & 89.1 \\
\hline $\begin{array}{l}\text { Terminal } \\
\text { voltageU/V }\end{array}$ & 14.12 & 13.72 & 12.53 & 12.32 & 13.79 \\
\hline $\begin{array}{l}\text { Residual } \\
\text { Capacityq/Ah }\end{array}$ & 20.21 & 16.89 & 6.61 & 4.79 & 17.28 \\
\hline Sample No. & 6 & 7 & 8 & 9 & 10 \\
\hline SOH\% & 89.1 & 89.0 & 83.1 & 83.1 & 82.6 \\
\hline Terminal & & & & & \\
voltageU/V & 13.63 & 12.41 & 14.61 & 13.59 & 12.49 \\
\hline
\end{tabular}




\begin{tabular}{|l|c|c|c|c|c|}
\hline $\begin{array}{l}\text { Residual } \\
\text { Capacityq/Ah }\end{array}$ & 15.69 & 6.22 & 17.81 & 13.41 & 6.78 \\
\hline
\end{tabular}

algorithm, the prediction results is shown in Table 5, the predicted curve is shown in Figure 3, the error is very small, the measuring accuracy is high, Results is satisfactory.

Table 5 predicted results and error analysis

\begin{tabular}{|c|c|c|c|c|c|}
\hline Sample No. & 1 & 2 & 3 & 4 & 5 \\
\hline $\begin{array}{c}\text { Predicted } \\
\text { results }\end{array}$ & 20.18 & 16.66 & 6.54 & 4.83 & 17.36 \\
\hline Error \% & 0.15 & 1.36 & 1.05 & 0.83 & 0.46 \\
\hline Sample No. & 6 & 7 & 8 & 9 & 10 \\
\hline $\begin{array}{c}\text { Predicted } \\
\text { results }\end{array}$ & 15.75 & 6.21 & 18.78 & 13.38 & 6.81 \\
\hline Error \% & 0.38 & 0.16 & 0.54 & 0.22 & 0.44 \\
\hline
\end{tabular}

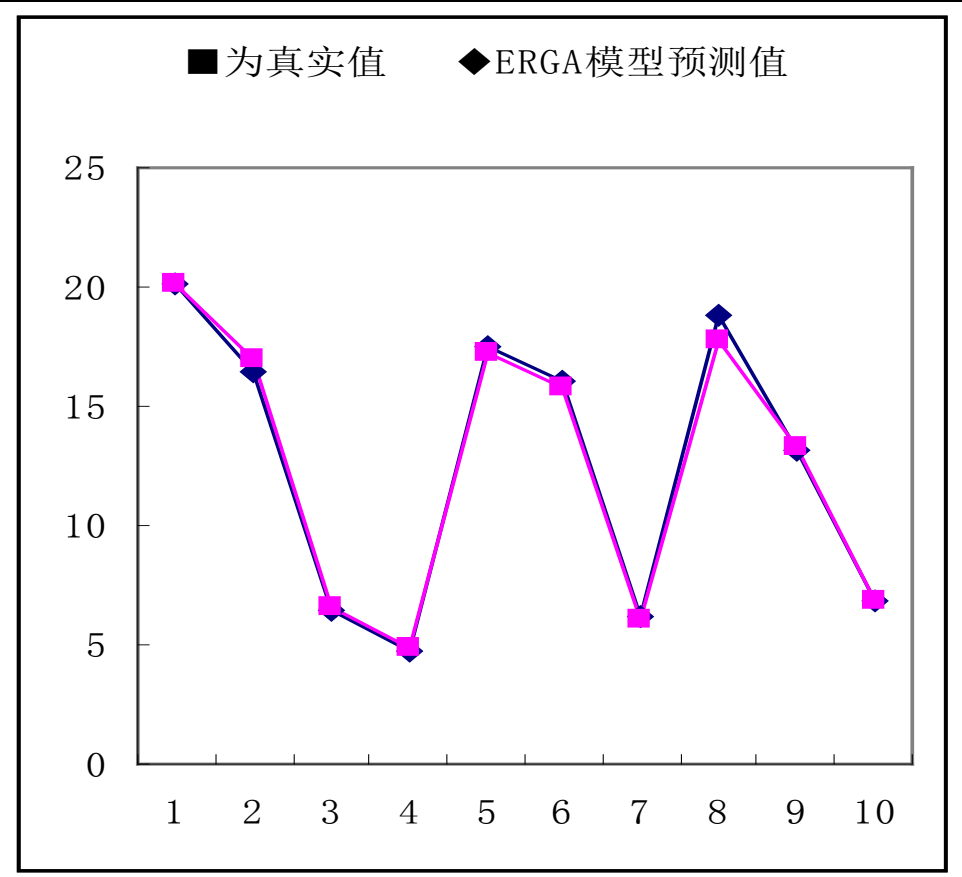

Figure 3 RS-SVM predicted results based on ERGA

\section{Conclusions}

In the paper, the method of combining rough set with support vector machine to predict battery SOC is adept ,By the improved reduction algorithm based on mutual information heuristic attribute , reduce system data, Minimum attribute reduction set is classified by SVM.The experimental and predicted results show that the forecast effect applied in the terminal voltage, $\mathrm{SOH} \%$ and residual capacity is good, The RS-SVM method has important research value.

\section{Acknowledgements}

National Technical Innovation Fund for small and medium enterprises(12C26212201322),

Strategic emerging industry scientific and technological innovation projects (2013121005) 


\section{References}

[1] Dj.M. Maric, P.F. Meier and S.K. Estreicher: Mater. Sci. Forum Vol. 83-87 (1992), p. 119

[2] M.A. Green: High Efficiency Silicon Solar Cells (Trans Tech Publications, Switzerland 1987).

[3] Y. Mishing, in: Diffusion Processes in Advanced Technological Materials, edtied by D. Gupta Noyes Publications/William Andrew Publising, Norwich, NY (2004), in press.

[4] G. Henkelman, G.Johannesson and H. Jónsson, in: Theoretical Methods in Condencsed Phase Chemistry, edited by S.D. Schwartz, volume 5 of Progress in Theoretical Chemistry and Physics, chapter, 10, Kluwer Academic Publishers (2000).

[5] R.J. Ong, J.T. Dawley and P.G. Clem: submitted to Journal of Materials Research (2003)

[6] P.G. Clem, M. Rodriguez, J.A. Voigt and C.S. Ashley, U.S. Patent 6,231,666. (2001)

[7]Eman,O.E., Interactivebi-level multi-objective integer non-linear programming problem. Applied Mathematical Sciences,5(65),3221-3232,2011. 